\title{
Turbulence Spectra from Doppler-shifted Spectral Lines
}

\author{
A. Lazarian \\ Department of Astronomy, University of Wisconsin-Madison, lazarian@astro.wisc.edu
}

\begin{abstract}
Turbulence is a key element of the dynamics of astrophysical fluids, including those of interstellar medium, clusters of galaxies and circumstellar regions. Turbulent motions induce Doppler shifts of observable emission and absorption lines. In the review we discuss new techniques that relate the spectra of underlying velocity turbulence and spectra of Doppler-shifted lines. In particular, the Velocity-Channel Analysis (VCA) makes use of the channel maps, while the Velocity Coordinate Spectrum (VCS) utilizes the fluctuations measured along the velocity axis of the Position-Position Velocity (PPV) data cubes. Both techniques have solid foundations based on analytical calculations as well as on numerical testings. Among the two the VCS, which has been developed quite recently, has two advantages. First of all, it is applicable to turbulent volumes that are not spatially resolved. Second, it can be used with absorption lines that do not provide good spatial sampling of different lags over the image of turbulent object. In fact, numerical testing shows that measurements of Doppler shifted absorption lines over a few directions is sufficient for a reliable recovering of the underlying spectrum of the turbulence. Our comparison of the VCA and the VCS with a more traditional technique of Velocity Centroids, shows that the former two techniques recover reliably the spectra of supersonic turbulence, while the Velocity Centroids may have advantages for studying subsonic turbulence. In parallel with theoretical and numerical work on the VCA and the VCS, the techniques have been applied to spectroscopic observations. We discuss results on astrophysical turbulence obtained with the VCA and the VCS.
\end{abstract}

\section{WHAT CAN TURBULENT SPECTRA TELL US?}

As a rule astrophysical fluids are turbulent and the turbulence is magnetized. This ubiquitous turbulence determines the transport properties of interstellar medium (see Elmegreen \& Falgarone 1996, Stutzki 2001, Balesteros-Peredes et al. 2006) and intracluster medium (see Sunyaev, Norman \& Bryan 2003, Ensslin \& Vogt 2006, Lazarian 2006), many properties of Solar and stellar winds (see Hartman \& McGregor 1980) etc. One may say that to understand heat conduction, propagation of cosmic rays and electromagnetic radiation in different astrophysical environments it is absolutely essential to understand the properties of underlying magnetized turbulence. The fascinating processes of star formation (see McKee \& Tan 2002, Elmegreen 2002, Mac Low \& Klessen 2004) and interstellar chemistry ( see Falgarone et al. 2006 and references therein) are also intimately related to properties of magnetized compressible turbulence (see reviews by Elmegreen \& Scalo 2004).

From the point of view of fluid mechanics astrophysical turbulence is characterized by huge Reynolds numbers, $R e$, which is the inverse ratio of the eddy turnover time of a parcel of gas to the time required for viscous forces to slow it appreciably. For $R e \gg 100$ we expect gas to be turbulent and this is exactly what we observe in HI 
(for HI $R e \sim 10^{8}$ ). In fact, very high astrophysical $R e$ and its magnetic counterpart magnetic Reynolds number $R m$ (that can be as high as $R m \sim 10^{16}$ ) present a big problem for numerical simulations that cannot possibly get even close to the astrophysicallymotivated numbers. The currently available 3D simulations can have $R e$ and $R m$ up to $\sim 10^{4}$. Both scale as the size of the box to the first power, while the computational effort increases as the fourth power ( 3 coordinates + time), so the brute force approach cannot begin to resolve the controversies related to ISM turbulence. This caused serious concerns that while present codes can produce simulations that resemble observations, whether numerical simulations reproduce reality well (see McKee 1999, Shu et al. 2004). We believe that these concerns may be addressed via observational studies of astrophysical turbulence.

Statistical description is a nearly indispensable strategy when dealing with turbulence. The big advantage of statistical techniques is that they extract underlying regularities of the flow and reject incidental details. Kolmogorov description of unmagnetized incompressible turbulence is a statistical one. For instance it predicts that the difference in velocities at different points in turbulent fluid increases on average with the separation between points as a cubic root of the separation, i.e. $|\delta v| \sim l^{1 / 3}$. In terms of direction-averaged energy spectrum this gives the famous Kolmogorov scaling $E(k) \sim 4 \pi k^{2} P(\mathbf{k}) \sim k^{5 / 3}$, where $P(\mathbf{k})$ is a $3 D$ energy spectrum defined as the Fourier transform of the correlation function of velocity fluctuations $\xi(\mathbf{r})=\langle\delta v(\mathbf{x}) \delta v(\mathbf{x}+\mathbf{r})\rangle$. Note that in this paper we use $\langle\ldots\rangle$ to denote averaging procedure.

The example above shows the advantages of the statistical approach to turbulence. For instance, the energy spectrum $E(k) d k$ characterizes how much energy resides at the interval of scales $k, k+d k$. At large scales $l$ which correspond to small wavenumbers $k$ ( i.e. $l \sim 1 / k$ ) one expects to observe features reflecting energy injection. At small scales one should see the scales corresponding to sinks of energy. In general, the shape of the spectrum is determined by a complex process of non-linear energy transfer and dissipation.

In view of the above it is not surprising that attempts to obtain spectra of interstellar turbulence have been numerous since 1950s (see Munch 1958). However, various directions of research achieved various degree of success (see Armstrong, Rickett \& Spangler 1995). For instance, studies of turbulence statistics of ionized media were more successful (see Spangler \& Gwinn 1990) and provided the information of the statistics of plasma density at scales $10^{8}-10^{15} \mathrm{~cm}$. However, these sort of measurements provide only the density statistics, which is an indirect measure of turbulence.

Velocity statistics is much more coveted turbulence measure. Although, it is clear that Doppler broadened lines are affected by turbulence, recovering of velocity statistics was extremely challenging without an adequate theoretical insight. Indeed, both velocity and density contribute to fluctuations of the intensity in the Position-Position-Velocity (PPV) space. In what follows we discuss how the observable Doppler-shifted lines can be used to recover a spectrum of turbulent velocity using two new techniques, that, unlike other mostly empirical techniques, have solid theoretical foundations. How to obtain using spectroscopic observations other characteristics of turbulence, e.g. higher order statistics, anisotropies has been reviewed earlier (see Lazarian 2004). 

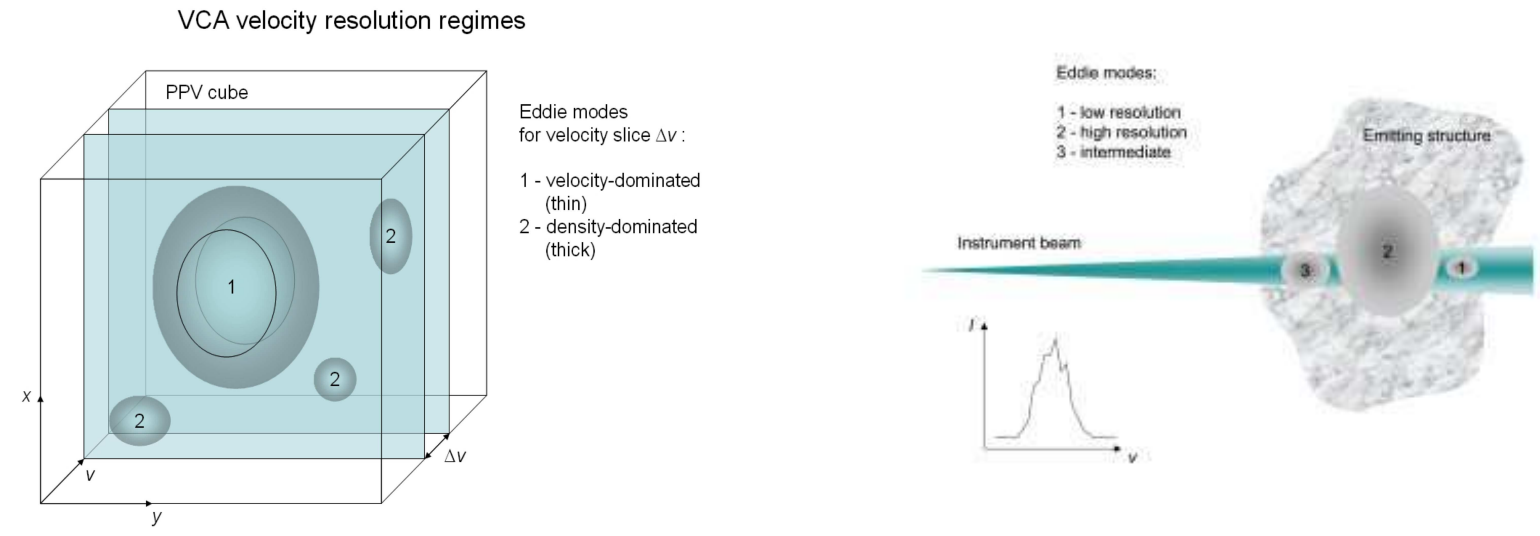

FIGURE 1. Left Panel: PPV data cube. Illustration of the concepts of the thick and thin velocity slices. the slices are thin for the PPV images of the large eddies, but thick for the images of small eddies. Right Panel: Illustration of the VCS technique. For a given instrument resolution large eddies are in the high resolution limit, while small eddies are in the low resolution limit.

\section{VCA AND VCS: TWO WAYS TO ANALYZE SPECTRAL DATA}

Spatial spectra obtained by taking Fourier transform of channel maps had been widely used to study HI before we conducted our theoretical study of what those spectra mean in Lazarian \& Pogosyan (2000, henceforth LP00). The channel maps correspond to the velocity slices of PPV cubes as shown in Figure 1 and one may naturally ask a question whether anything depends on the thickness of the channel. It is intuitively clear that if medium is optically thin and the velocity is integrated over the whole spectral line, the fluctuations can depend only on density inhomogeneities. It is also suggestive that the contribution of the velocity fluctuations may depend on whether the images of the eddies under study fit within a velocity slice or their velocity extend is larger than the slice thickness (see Figure 1). According to LP00 this results in the assymptotics that correspond to "thin" and "thick" velocity channels. Note, that these questions were not posed by the earlier research. This resulted in channel map spectra with different spectral indexes the relation of which to the underlying velocity fluctuations was unclear (see Green 1990 and references therein).

Some spectral data, for instance, optically thick CO data were traditionally analyzed in differently (see Falgarone \& Puget 1995), namely, spectra of total intensities was studied. The origin of this spectrum and its relation to the underlying velocity and density fluctuations was established in Lazarian \& Pogosyan (2004, henceforth LP04). This work also clarified the effects of absorption that were reported for HI data. In terms of the techniques of turbulence study LP04 deals with the VCA, but in the case when absorption is present.

A radically different way of analyzing spectroscopic data is presented in Lazarian \& Pogosyan (2006, henceforth LP06) and Chepurnov \& Lazarian (2006a). There the spectra along the V-axis of the PPV cube are studied (see Figure 2). The technique was 
termed Velocity Coordinate Spectrum (VCS) in Lazarian (2004). The formalism can be traced to LP00 work, where the expressions that relate the spectrum of fluctuations along the velocity coordinate and the underlying velocity spectrum were obtained. However, it took some time to understand the advantages that the VCS provides for the practical handling of the emission and absorption data. The first analysis of the data using the VCS is performed by Chepurnov \& Lazarian (in preparation). Numerical testing of the technique is provided in Chepurnov \& Lazarian (2006b).

\section{BASICS OF THE FORMALISM}

Below we provide a brief introduction to the mathematical foundations of the VCA and the VCS (see more in LP00, LP04 and LP06). Our goal is to relate the statistics that can be obtained through spectral line observations, for instance, the structure function of the intensity of emission $I_{\mathbf{X}}(v)$

$$
\mathscr{D}\left(\mathbf{X}, v_{1}, v_{2}\right) \equiv\left\langle\left[I_{\mathbf{X}}\left(v_{1}\right)-I_{\mathbf{X}}\left(v_{2}\right)\right]^{2}\right\rangle,
$$

where the $z$-axis component velocity $v$ is measured by in the direction defined by the two dimensional vector ${ }^{1} \mathbf{X}$, to the underlying properties of the turbulent cascade.

The intensities $I_{\mathbf{X}}(v)$ are affected by both the turbulence and the absorption. To quantify these effects we consider the standard equation of radiative transfer $d I_{V}=$ $-g_{v} I_{v} d s+j_{v} d s$, where $g_{v}=\alpha(\mathbf{x}) \rho(\mathbf{x}) \phi_{v}(\mathbf{x}), j_{v}=\tilde{\varepsilon} \rho(\mathbf{x}) \phi_{v}(\mathbf{x}), \mathbf{x}$ is a three dimensional position vector $(\mathbf{X}, z), \rho(\mathbf{x})$ is the density and $\phi_{v}(\mathbf{x})$ is the velocity distribution of the atoms. The turbulent motions affect the velocity distribution. Indeed, for the line-ofsight component, $v$, of the velocity at the position $\mathbf{x}$, it is a sum of the $z$-components of the regular gas flow (e.g., due to galactic rotation) $v_{\text {gal }}(\mathbf{x})$, the turbulent velocity $u(\mathbf{x})$ and the residual component due to thermal motions. This residual thermal velocity $v-v_{g a l}(\mathbf{x})-u(\mathbf{x})$ has a Maxwellian distribution, so

$$
\phi_{v}(\mathbf{x}) \mathrm{d} v=\frac{1}{(2 \pi \beta)^{1 / 2}} \exp \left[-\frac{\left(v-v_{g a l}(\mathbf{x})-u(\mathbf{x})\right)^{2}}{2 \beta}\right] \mathrm{d} v,
$$

where $\beta=\kappa_{B} T / m, m$ being the mass of atoms. For $T \rightarrow 0$ the function $\phi_{v}$ tends to a delta-function that depends on regular gas flow and the turbulent velocity $u$. Taking Fourier transforms we deal with velocity gradients, which are larger for turbulent motions than for large-scale sheer. For instance, the latter for the Galactic rotation is given by the Oort's constant, which is $14 \mathrm{~km} \mathrm{~s}^{-1} \mathrm{kpc}^{-1}$. In comparison, the shear due to typical Kolmogorov-type turbulent motions in the Galaxy with the injection of energy at $10 \mathrm{~km} \mathrm{~s}^{-1}$ at the scale of $L \sim 30 \mathrm{pc}$ is $\sim 300 \mathrm{~km} \mathrm{~s}^{-1}(L / l)^{2 / 3} \mathrm{kpc}^{-1}$. Thus, in spite of the fact, that regular large-scale galactic shear velocities may be much larger than the

\footnotetext{
${ }^{1}$ Henceforth we denote by the capital bold letters the two dimensional position-position vectors that specify the line of sight. Small bold letters are reserved to describe the vectors of three dimensional spatial position. The $z$-axis is chosen to be along the line of sight.
} 
turbulent velocities, they can be neglected for our analysis (LP00 and a numerical study in Esquivel et al. 2003).

For the $z$-component of the turbulent velocity field (i.e. $u$ ) we use the structure function

$$
D_{z}(\mathbf{r})=\left\langle(u(\mathbf{x}+\mathbf{r})-u(\mathbf{x}))^{2}\right\rangle,
$$

which for a self-similar power-law turbulent motions provide $D_{z} \sim r^{m}$, where $m=1 / 3$ for Kolmogorov turbulence. These velocity correlations together with the correlations of over-density

$$
\xi(r)=\xi(\mathbf{r})=\langle\rho(\mathbf{x}) \rho(\mathbf{x}+\mathbf{r})\rangle,
$$

enter the correlation function that can be constructed from the PPV densities $\rho_{s}$, which are available through spectroscopic observations. If the gas is confined in an isolated cloud of size $S$ and the galactic shear over this scale is neglected, the zero-temperature correlation function is (see LP06)

$$
\xi_{s}(R, v) \equiv\left\langle\rho_{S}\left(\mathbf{X}_{1}, v_{1}\right) \rho\left(\mathbf{X}_{2}, v_{2}\right)\right\rangle \propto \int_{-S}^{S} \mathrm{~d} z\left(1-\frac{|z|}{S}\right) \frac{\xi(r)}{D_{z}^{1 / 2}(\mathbf{r})} \exp \left[-\frac{v^{2}}{2 D_{z}(\mathbf{r})}\right],
$$

where the correlation function $\xi_{s}$ is defined in the PPV space, where $R$ is the spatial separation between points in the plane-of-sky and $v$ is the separation along the $\mathrm{V}$-axis.

TABLE 1. The short-wave asymptotical behavior of power spectra in PPV space. $\gamma$ is the power-law index of the density correlation function, $m$ is the index of the velocity correlation function. The spectrum of fluctuations in the PPV volume can be presented as the sum of $P_{\rho}$, which has both density and velocity contributions and $P_{v}$, which has only the contribution arising from turbulent velocity. From LP06.

\begin{tabular}{cccc}
\hline & $1 \mathrm{D}: P_{S}\left(k_{v}\right)$ & $2 \mathrm{D}: P_{S}(K)$ & $3 \mathrm{D}: P_{S}\left(K, k_{v}\right)$ \\
$k_{v} D_{z}^{1 / 2}(S) \gg 1$ & $K S \gg 1$ & $k_{v}^{2} D_{z}(S) \gg(k S)^{m}$ \\
\hline$P_{\rho}:$ & $\left(r_{0} / S\right)^{\gamma}\left[k_{v} D_{z}^{1 / 2}(S)\right]^{2(\gamma-1) / m}$ & $\left(r_{0} / S\right)^{\gamma}[K S]^{\gamma+m / 2-3}$ & $\left(r_{0} / S\right)^{\gamma}\left[k_{v} D_{z}^{1 / 2}(S)\right]^{-2(3-\gamma) / m}$ \\
\hline$P_{v}:$ & {$\left[k_{v} D_{z}^{1 / 2}(S)\right]^{-2 / m}$} & {$[K S]^{m / 2-3}$} & {$\left[k_{v} D_{z}^{1 / 2}(S)\right]^{-6 / m}$} \\
\hline
\end{tabular}

The correlation function of over-density given by Eq. (4) has a constant part that depends on the mean density only and the part that changes with $r$. For instance, for the power-law density spectrum the correlation functions of over-density take the form (see LP06 for the discussion of cases of $\gamma<0$ and $\gamma>0$ ):

$$
\xi(r)=\langle\rho\rangle^{2}\left(1+\left[\frac{r_{0}}{r}\right]^{\gamma}\right)
$$

where $r_{0}$ has the physical meaning of the scale at which fluctuations are of the order of the mean density (see more in LP06). Substituting Eq. (6) in Eq. (5) it is easy to see that 
TABLE 2. Conditions for the impact of density inhomogeneities to the PPV statistics exceeds the velocity contribution. $\gamma$ must be larger than 0 .

$$
\begin{array}{ll}
\hline m \geq \max \left[\frac{2}{3}, \frac{2}{3}(1-\gamma)\right] & v^{2}<D_{z}(S)\left(r_{0} / S\right)^{m} \\
\frac{2}{3}(1-\gamma)<m<\frac{2}{3} & v^{2}<D_{z}(S)\left(r_{0} / S\right)^{\frac{2 / 3 \gamma m}{m-2 / 3(1-\gamma)}} \\
m \leq \min \left[\frac{2}{3}, \frac{2}{3}(1-\gamma)\right] & r_{0} / S>1
\end{array}
$$

TABLE 3. The VCA assymptotics. Thin means that the channel width $<$ velocity dispersion at the scale under study; thick means that the channel width $>$ velocity dispersion at the scale under study; very thick means that a substantial part of the velocity profile is integrated over.

\begin{tabular}{lcc}
\hline Slice & Shallow 3-D density & Steep 3-D density \\
thickness & $P_{n} \propto k^{-3+\gamma}, \gamma>0$ & $P_{n} \propto k^{-3+\gamma}, \gamma<0$ \\
\hline 2-D intensity spectrum for thin slice & $\propto K^{-3+\gamma+m / 2}$ & $\propto K^{-3+m / 2}$ \\
2-D intensity spectrum for thick slice & $\propto K^{-3+\gamma}$ & $\propto K^{-3-m / 2}$ \\
2-D intensity spectrum for very thick slice & $\propto K^{-3+\gamma}$ & $\propto K^{-3+\gamma}$ \\
\hline
\end{tabular}

the PPV correlation function $\xi_{s}$ can be presented as a sum of two terms, one of which does depend on the fluctuations of density, the other does not. Taking Fourier transform of $\xi_{s}$ one gets the PPV spectrum that evidently is also a sum of two terms $P_{\rho}$ and $P_{v}$ the assymptotics for which are presented in Table 1.

Expression (5) and its generalizations may be used directly to solve the inverse problem to find the properties of the underlying astrophysical turbulence for an arbitrary spectrum. However, so far, most attention was given to astrophysically important case of power-law turbulence. Note, that using spectra rather than the correlation function has advantages. For instance, the correlations along the V-axis of the PPV cube may be dominated by large-scale gradients, while spectra provide correct result (see explanation in LP04 and LP06). The results for 1, 2 and 3 dimensional spectra are presented in Table 1 . In many cases the contributions that depend on density are subdominant. Table 2 presents special cases when density fluctuations are important.

\section{VELOCITY CHANNEL ANALYSIS}

The interpretation of the channel maps is the domain of the VCA. Table 3 shows how the power spectrum of the intensity fluctuations depends on the thickness of the velocity channel. Below we provide quantitative discussion of the VCA.

It is easy to see that both steep and shallow underlying density the power law index steepens with the increase of velocity slice thickness. In the thickest velocity slices the velocity information is averaged out and we get the density spectral index $-3+\gamma$. The velocity fluctuations dominate in thin slices, and the index $m$ that characterizes the velocity fluctuation can be obtained using thin velocity slices (see Table 1). As we mentioned earlier, the notion of thin and thick slices depends on a turbulence scale under study and the same slice can be thick for small scale turbulent fluctuations and thin for 
large scale ones (see Figure 1).

One may notice that the spectrum of intensity in a thin slice gets shallower as the underlying velocity get steeper. To understand this effect let us consider turbulence in an incompressible optically thin medium. The intensity of emission in a velocity slice is proportional to the number of atoms per the velocity interval given by the thickness of the slice. Thin slice means that the velocity dispersion at the scale of study is larger than the thickness of a slice. The increase of the velocity dispersion at a particular scales means that less and less energy is being emitted within the velocity interval that defines the slice. As the result the PPV image of the eddy gets fainter. In other words, the larger is the dispersion at the scale of the study the less intensity is registered at this scale within the thin slice of spectral data. This means that steep velocity spectra that correspond to the flow with more energy at large scales should produce intensity distribution within thin slice for which the more brightness will be at small scales. This is exactly what our formulae predict for thin slices (see Table 3).

If density variations are also present they modify the result. For small scale asymptotics of thin slices this happens, however, only when the density spectrum is shallow (i.e. $\gamma>0$ ), i.e. dominated by fluctuations at small scales (see Eq. (6)).

\section{VELOCITY COORDINATE SPECTRUM}

The VCS is a brand new technique, which, unlike the VCA, was not motivated by the interpretation of the existing observations. In the case of the VCS it were theoretical advances that induced the subsequent data analysis.

Unlike the standard spatial spectra, that are functions of angular wavenumber, the VCS is a function of the wave number $k_{v} \sim 1 / v$, which means that large $k_{v}$ correspond to small velocity differences, while small $k_{v}$ correspond to large velocity differences.

A realistic beam has a finite width, $\Delta B$. We remind the reader that we deal with the case in which the turbulent volume extend along the line of sight $S$ is much smaller than the distance to the volume. As the result, the angular extend of the beam is straightforwardly related to the physical scales that we deal with. The VCS near a scale $k_{v}$ depends on whether the instrument resolves the correspondent spatial scale $\left[k_{v}^{2} D_{z}(S)\right]^{-1 / m} S$. If this scale is resolved then $P_{v}\left(k_{v}\right) \propto k_{v}^{-2 / m}$ and $P_{\rho}\left(k_{v}\right) \propto k_{v}^{-2(1-\gamma) / m}$. If the scale is not resolved then $P_{v}\left(k_{v}\right) \propto k_{v}^{-6 / m}$ and $P_{\rho}\left(k_{v}\right) \propto k_{v}^{-2(3-\gamma) / m}$. These results are presented in a compact form in Table 4. The transition from the low to the high resolution regimes happens as the velocity scale under study gets comparable to the turbulent velocity at the minimal spatially resolved scale. As the change of slope is the velocity-induced effect, it is not surprising that the difference in spectral indexes in the low and high resolution limit is $4 / m$ for both $P_{v}$ and $P_{\rho}$ terms, i.e it does not depend on the density ${ }^{2}$. This allows for separation of the velocity and density contributions.

\footnotetext{
${ }^{2}$ In the situation where the available telescope resolution is not sufficient, i.e. in the case of extragalactic turbulence research, the high spatial resolution VCS can be obtained via studies of the absorption lines from point sources.
} 

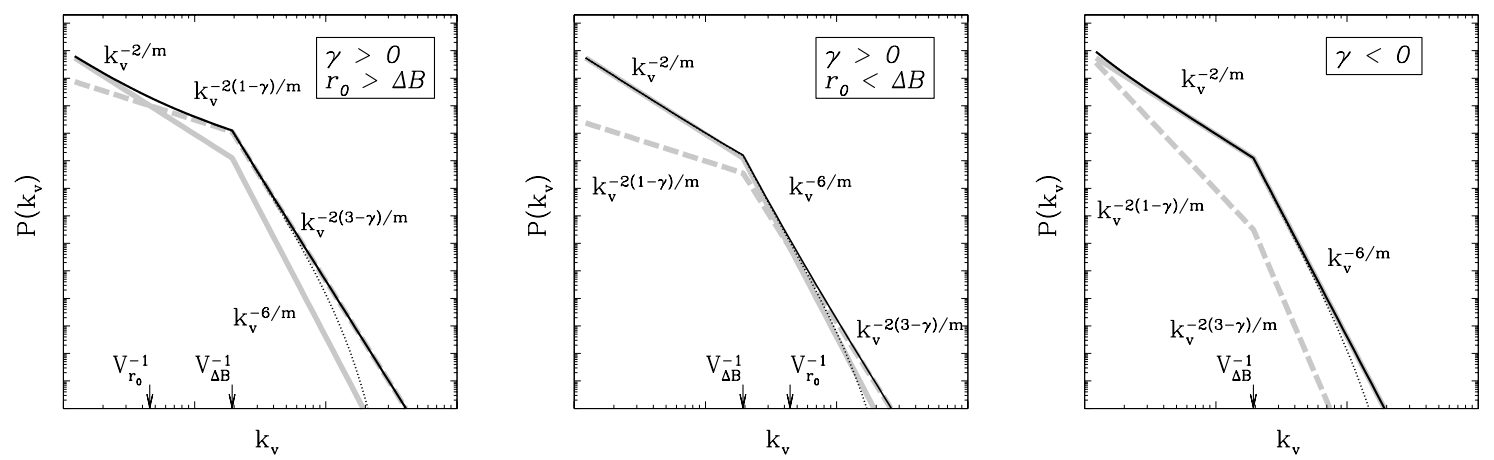

FIGURE 2. Particular cases for the VCS (from LP06). In every panel light lines show contributions from the $\rho$-term (density modified by velocity, dashed line) and $v$-term (pure velocity effect, solid line) separately, while the dark solid line shows the combined total VCS power spectrum. Thermal suppression of fluctuations is shown by the dotted line. Labels below the dark solid lines mark the scaling of the subdominant contributions. For the left and middle panels the density power spectrum is taken to be shallow, $\gamma>0$. The left panel corresponds to high amplitude of the density correlations, $r_{0}>\Delta B$, where density effects become dominant at relatively long wavelengths for which the beam is narrow. In the middle panel, the amplitude of density correlations is low $r_{0}<\Delta B$ and they dominate only the smallest scales which results in the intermediate steepening of the VCS scaling. The right panel corresponds to the steep density spectrum. In this case the density contribution is always subdominant. In this example the thermal scale is five times shorter than the resolution scale $V_{\Delta B}$.

TABLE 4. Scalings of VCS for shallow and steep densities (from LP06). To the linear scale $\Delta B$ corresponds the velocity scale $V_{\Delta B} \equiv$ $\sqrt{D(S)(\Delta B / S)^{m}}$, equal to the magnitude of turbulent velocities at the separation of a size $\Delta B$. It is not difficult to find that when $k_{v}^{-1}>V_{\Delta B}$ the beam is narrow, while on smaller scales its width is important.

\begin{tabular}{lll}
\hline Spectral term & $\Delta B<S\left[k_{v}^{2} D_{z}(S)\right]^{-\frac{1}{m}}$ & $\Delta B>S\left[k_{v}^{2} D_{z}(S)\right]^{-\frac{1}{m}}$ \\
\hline$P_{\rho}\left(k_{v}\right)$ & $\propto\left(k_{v} D_{z}^{1 / 2}(S)\right)^{-2(1-\gamma) / m}$ & $\propto\left(k_{v} D_{z}^{1 / 2}(S)\right)^{-2(3-\gamma) / m}$ \\
\hline$P_{v}\left(k_{v}\right)$ & $\propto\left(k_{v} D_{z}^{1 / 2}(S)\right)^{-2 / m}$ & $\propto\left(k_{v} D_{z}^{1 / 2}(S)\right)^{-6 / m}$
\end{tabular}

For instance, Figure 2 illustrates that in the case of shallow density both the density and velocity spectra can be obtained. Potentially, procedures of extracting the information on the 3D turbulent density can be developed for the steep density case as well. However, this requires careful accounting for errors that arise while the major contribution arising 

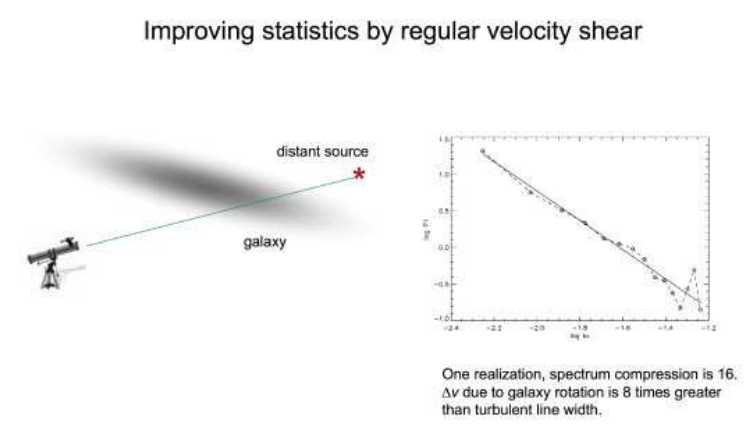

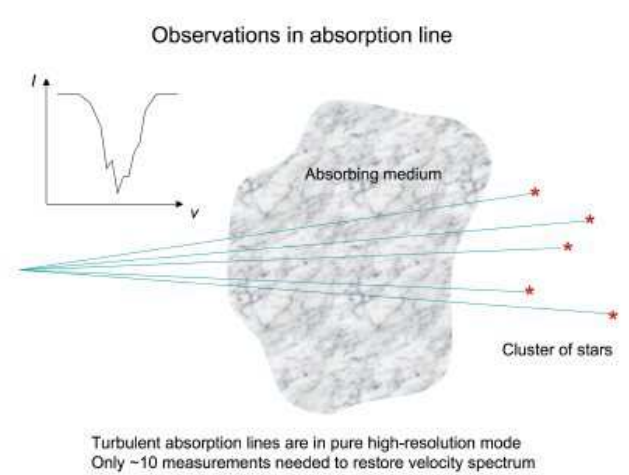

Turbulent absorption lines are in pure high-resolution mode
Only -10 measurements needed to restore velocity spectrum

FIGURE 3. Illustration of VCS absorption studies of turbulence. Left Panel: In case an external galaxy in which regular shear within the galaxy helps to improve the statistics. Right Panel: For a cloud we showed that we can restore the turbulence spectrum with spectroscopic measurements for less than 10 directions. From Chepurnov \& Lazarian 2006b

from velocity is subtracted from the data ${ }^{3}$.

The VCS is applicable to both emission and absorption studies of turbulence. A numerical study in Chepurnov \& Lazarian (2006b) shows that having just several lines of sight along which the absorption spectra is measured is sufficient for recovering the underlying turbulent velocity spectrum (see Figure 3). In the case of strong regular shear just one line of sight may be sufficient.

\section{EFFECTS OF ABSORPTION}

The issues of absorption were worrisome for the researchers from the very start of the research in the field (see Munch 1958). The erroneous statements about the effects of absorption on the observed turbulence statistics are widely spread in the literature (see discussion in LP04).

Using transitions that are less affected by absorption, e.g. HI, may allow to avoid the problem. However, it looks regretful not to use the wealth of spectroscopic data only because absorption may be present. A study of absorption effects in LP04 and LP06. For the VCA it was found that for sufficiently thin ${ }^{4}$ slices the scalings obtained in the absence of absorption still hold provided that the absorption on the scales under

\footnotetext{
${ }^{3}$ Needless to say, when the turbulent object is resolved, the easiest way to obtain the density spectral index is to study the integrated intensity maps, provided that the absorption is negligible (see criteria for this in LP04).

${ }^{4}$ The thermal broadening limits to what extend the slice can be thin. This means that in some cases that the actual turbulent velocity spectrum may not be recoverable.
} 
study is negligible. The criterion for the absorption to be important is $\alpha^{2}\left\langle\left(\rho_{s}\left(\mathbf{X}, v_{1}\right)-\right.\right.$ $\left.\left.\rho_{s}\left(\mathbf{X}, v_{2}\right)\right)^{2}\right\rangle \sim 1$, which for $\gamma<0$ results in the critical size of the slice thickness $V_{c}$ given by (LP06)

$$
\begin{aligned}
& V_{c} / D_{z}(S)^{1 / 2} \approx\left(\alpha \bar{\rho}_{s}\right)^{\frac{2 m}{m-2}}, \quad m>2 / 3 \\
& V_{c} / D_{z}(S)^{1 / 2} \approx\left(\alpha \bar{\rho}_{s}\right)^{-1}, \quad m<2 / 3,
\end{aligned}
$$

where $\bar{\rho}_{s}$ is the mean PPV density. The absorption is dominant for the slices thicker than $V_{c}$. The difference with the case of $\gamma>0$ is that in the latter case one should also consider a possibility that density contribution can be important (see Table 2). The criterion above coincides with one for the VCS, if we identify the critical $k_{v}$ with $1 / V_{c}$. If the resolution of the of the telescope is low, another limitation applies. The resolved scale should be less than the critical spatial scale that arises from the condition

$\alpha^{2}\left\langle\left(\rho_{s}\left(\mathbf{X}_{1}, v\right)-\rho_{s}\left(\mathbf{X}_{2}, v\right)\right)^{2}\right\rangle \sim 1$ which for $\gamma<1$ results in $R_{c} / S \approx\left(\alpha \bar{\rho}_{s}\right)^{\frac{2}{m-2}}$ (LV06). If only scales larger than $R_{c}$ are resolved, the information on turbulence is lost.

If integrated intensity of spectral lines is studied in the presence of absorption nontrivial effects emerge. Indeed, for optically thin medium the spectral line integration results in PPV intensity fluctuations that reflect the density statistics. LP04 showed that this may not be any more true for lines affected by absorption. When velocity is dominant a very interesting regime for which intensity fluctuations show universal behavior, i.e. the power spectrum $P(K) \sim K^{-3}$ emerges. When density is dominant (see Table 2), the spectral index of intensity fluctuations in those two situations is the same as in the case an optically thin cloud integrated through its volume. This means that for $\gamma>0$ in the range of parameter space defined by Table 2 the measurements of intensity fluctuations of the integrated spectral lines reflect the actual underlying density spectrum in spite of the absorption effects.

\section{COMPARISON OF TECHNIQUES}

Traditionally the techniques to study velocity turbulence, e.g. velocity centroids or VCA, require the observations to spatially resolve the scale of the turbulence under study ${ }^{5}$. This constrains the variety of astrophysical objects where the turbulence can be studied. In this way, the VCS, is a unique tool that allows studies of astrophysical turbulence even when the instrument does not resolve the turbulent fluctuations spatially.

Our study of the effect of finite temperatures for the technique reveals that, unlike the VCA, the temperature broadening does not prevent the turbulence spectrum from being recovered from observations. Indeed, in VCA, gas temperature acts in the same way as the width of a channel. Within the VCS the term with temperature gets factorized and

\footnotetext{
${ }^{5}$ As it was discussed in LP00, the VCA can be applied directly to the raw interferometric data, rather than to images that require good coverage of all spatial frequencies. However, even with interferometers, the application of the VCA to extragalactic objects is restricted.
} 
it influences the amplitude of fluctuations (LP06). One can correct for this term ${ }^{6}$, which also allows for a new way of estimating the interstellar gas temperature.

Another advantage of the VCS compared to the VCA is that it reveals the spectrum of turbulence directly, while within the VCA the slope of the spectrum should be inferred from varying the thickness of the channel. As the thermal line width acts in a similar way as the channel thickness, additional care (see LP04) should be exercised not to confuse the channel that is still thick due to thermal velocity broadening with the channel that shows the thin slice asymptotics. A simultaneous use of the VCA and the VCS makes the turbulence spectrum identification more reliable.

The introduction of absorption in VCS and VCA brings about different results. Within the analysis of velocity slices spectra (VCA) the absorption results in new scalings for slices for which absorption is important. The turbulence spectral indexes can be recovered for the VCA within sufficiently thin slices, provided that the thickness of the slices exceeds the thermal line width. For the VCS when absorption becomes important the spectra get exponentially damped. This simplifies the interpretation of the data.

Both VCA and VCS are applicable to studies of not only emission, but also absorption lines. However, the necessity of using extended emission sources limits the extent of possible VCA studies of turbulence. This is not an issue for the VCS, for which absorption lines from point sources can be used (see Figure 3). Interestingly enough, in this case the VCS asymptotics for the high resolution limit should be used irrespectively of the actual beam size of the instrument. Note, that the VCA and the VCS are applicable to lines for which emission depends not only to first but also to the second power of density (see discussions in LP04, LP06).

A more traditional approach to turbulence studies includes velocity centroids, i.e. $S(\mathbf{X})=\int v_{z} \rho_{s}\left(\mathbf{X}, v_{z}\right) \mathrm{d} v_{z}$, where $\rho_{s}$ is the density of emitters in the PPV space. ${ }^{7}$. Analytical expressions for structure functions ${ }^{8}$ of centroids, i.e. $\left\langle\left[S\left(\mathbf{X}_{\mathbf{1}}\right)-S\left(\mathbf{X}_{\mathbf{2}}\right)\right]^{2}\right\rangle$ were derived in Lazarian \& Esquivel (2003). In that paper a necessary criterion for centroids to reflect the statistics of velocity was established. Esquivel \& Lazarian (2005) confirmed the utility of the criterion and revealed that for MHD turbulence simulations it holds for subsonic or slightly supersonic turbulence (see also Ossenkopf et al. 2006). This is in contrast to the VCA and the VCS that provide reliable ways to study supersonic turbulence.

VCA is related to the Spectral Correlation Functions (SCF) (see Padoan, Goodman \& Juvela 2003 and references therein). The latter technique also deals statistics of velocity slices. However if, ignoring the normalization, we write the informative part of the SCF in our notations as $\left\langle\left[\int d v\left(\rho_{s}\left(\mathbf{X}_{1}, v\right)-\rho_{s}\left(\mathbf{X}_{2}, \mathbf{v}\right)\right)^{2}\right]^{1 / 2}\right\rangle$ we see that we cannot commute the ()$^{1 / 2}$ and \langle\rangle operations. This is very unfortunate, as if it had been possible, we

\footnotetext{
6 To do this, one may attempt to fit for the temperature that would remove the exponential fall off in the spectrum of fluctuations along the velocity coordinate (Chepurnov \& Lazarian 2006a).

${ }^{7}$ Traditionally the definition of centroids includes a normalization by the integral of $\rho_{s}$. This, however does not substantially improve the statistics, but makes the analytical treatment very involved (Lazarian \& Esquivel 2003).

${ }^{8}$ Expressions for the correlation functions are straightforwardly related to those of structure functions. The statistics of centroids using correlation functions was used in a later paper by Levier (2004).
} 
the SCF would have corresponded to $\xi_{s}^{1 / 2}(R, 0)$ (see Eq. (5)) for which we have the analytical theory. With all the wealth of data analyzed using the SCF (see Padoan et al. 2003) it is very tempting to investigate what sort of errors this mathematically incorrect commuting entails. Alternatively, it is appealing to reanalyze the data in Padoan et al. 2003 using the VCA. It also seems interesting, although currently not feasible, to find the connections between the VCA and VCS and another statistical tool, namely, Principal Component Analysis (see Heyer \& Brunt 2004 and references therein).

In LP00, LP04, LP06 we used HI as an example of species to which the technique is to be applied. Using heavier species that have lower thermal Doppler width of spectral lines allows one to study turbulence up to smaller scales. In addition, we would like to stress that the VCS technique can be used at different wavelength. For instance, the Xray spectrometers with high spatial resolution can be used to study of turbulence in hot plasma. In particular, the potential of VCS is high for studies of turbulence in clusters of galaxies (cf. Sunyaev et al. 2003 and references therein). A simulated example of such a study with the future mission Constellation X is provided in Lazarian (2006).

Studies of turbulence in objects which are poorly resolved spatially is a natural avenue for the VCS applications. Interestingly enough, in this case one can combine the absorption line studies, which would provide the VCS for the pencil beam, i.e. for the high resolution, with the emission studies that would provide the VCS in the poor resolution limit. Potentially, both velocity and density spectra can be obtained this way.

The importance of this work goes beyond the actual recovery of the particular powerlaw indexes. First of all, the techniques can be generalized to solve the inverse problem to recover non-power law turbulence spectra. This may be important for studying turbulence at scales at which either injection or dissipation of energy happens. Such studies are important for identifying astrophysical sources and sinks of turbulent energy. Second, studies of the VCS transition from low resolution to high resolution regimes (see Figure 2) allows one to separate thermal and non-thermal contributions to the line-widths as it is discussed in LP06. This could both test the thermal correction that can be applied to extend the power-law into sub-thermal velocity range (see also Chepurnov \& Lazarian 2006b) and enable studies of temperature distribution of the gas in atomic clouds (cf. Heiles \& Troland, 2003).

\section{TESTING AND APPLYING TO OBSERVATIONS}

VCA predictions were tested in Lazarian et al. (2001), Esquivel et al. (2003) and in Chepurnov \& Lazarian (2006b) using synthetic maps obtained with simulated powerlaw data as well as with numerical compressible MHD simulations. Simulated data cubes allowed both density and velocity statistics to be measured directly. Then these data cubes were used to produce synthetic spectra which were analyzed using the VCA. As the result, the velocity and density statistics were successfully recovered ${ }^{9}$.

The VCA has been applied to several data sets already. One of the first applications

\footnotetext{
9 A recent study in Chepurnov \& Lazarian (2006b) explains why an attempted testing of the VCA in Miville-Deschenes et al. (2003) had inadequate resolution and therefore brought erroneous results.
} 


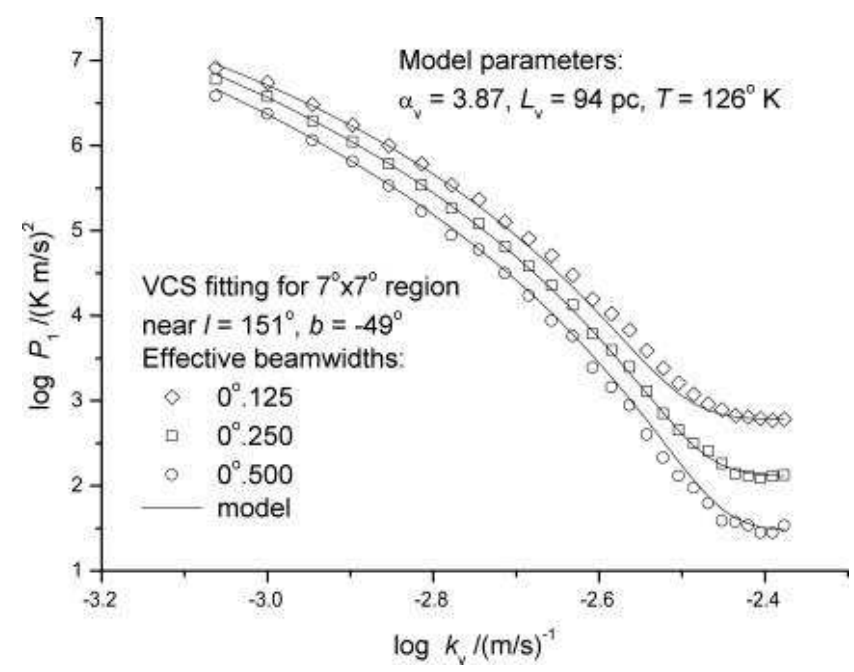

FIGURE 4. Application of the VCS to GALFA high latitude Arecibo data (by Chepurnov \& Lazarian). The spatial resolution of the maps was decreased to illustrate the VCS in both high and low resolution regimes. $\varepsilon$ here is the power spectrum index, which for the Kolmogorov turbulence is $11 / 3$. The measured $\varepsilon$ is in the range $[3.52 ; 3.57]$.

was in Stanimirovic \& Lazarian (2001), where the technique was applied to the Small Magellanic Cloud (SMC) data. The analysis revealed spectra of 3D velocity fluctuations roughly consistent with the Kolmogorov scaling (a bit more shallow). LP00 argued that the Kolmogorov scaling was expected for the magnetized turbulence appealing to the Goldreich-Shridhar (1995) model. Esquivel et al. (2003) used simulations of MHD turbulent flows to show that in spite of the presence of anisotropy caused by magnetic field the expected scaling of fluctuations is indeed Kolmogorov. Studies by Cho \& Lazarian (2003) revealed that the Kolmogorov-type scaling is also expected in the compressible MHD flows. This also supports the conclusion in LP00 that the data in Green (1993) is consistent with MHD turbulence scaling.

Muller et al. (2004) applied VCA to the Magellanic Bridge and discovered two structures with distinctly different properties. If the spectrum of turbulence in the southern part is similar to that in the SMC (velocity is roughly consistent with the Kolmogorov value and the density somewhat shallower the Kolmogorov value), the northern part of the Magellanic Bridge reveals completely different properties. If we interpret the results for the northern part using Table 3 the velocity power spectrum we get is very steep (index $\sim 4.2$ compared with Kolmogorov $\sim 3.7$ ), while the density is shallow (index $\sim 2.8$ ). Whether these values should be interpreted in terms of turbulence (some sort of shocks) or some other effects dominate is an open question that requires further studies.

Studies of turbulence are more complicated for the inner parts of the Galaxy, where (a) two distinct regions at different distances from the observer contribute to the emissivity for a given velocity and (b) effects of the absorption are important. However, the analysis in Dickey et al. (2001) showed that some progress may be made even in those unfavorable circumstances. Dickey et al. (2001) found the steepening of the spectral index with the increase of the velocity slice thickness. They also observed the spectral index for strongly absorbing direction approached -3 in accordance with the conclu- 
sions in LP04. Note, that the effects of optical depths may explain some other case when the spectral index stayed the same, e.g. -3 , while the thickness of the slice was varying (see Kralil et al. 2006). Incidently, this situation can be confused with the situation when the fluctuations arise from density only (see Begum et al. 2006).

$21-\mathrm{cm}$ absorption provides another way of probing turbulence on small scales. The absorption depends on the density to temperature ratio $\rho / T$, rather than to $\rho$ as in the case of emission ${ }^{10}$. However, in terms of the VCA this change is not important and we still expect to see emissivity index steepening as velocity slice thickness increases, provided that velocity effects are present. In view of this, results of Deshpande et al. (2001), who did not see such steepening, can be interpreted as the evidence of the viscous suppression of turbulence on the scales less than $1 \mathrm{pc}$. The fluctuations in this case should be due to density and their shallow spectrum $\sim k^{-2.8}$ may be related to the damped magnetic structures below the viscous cutoff (see Lazarian, Vishniac, \& Cho 2004). This may be also a consequence of the shallow density spectrum in compressible MHD (see Beresnyak, Lazarian \& Cho 2005).

Historically, the CO data was analyzed after integration over the entire emission line. Stutzki et al. (1998) presented the power spectra of ${ }^{12} \mathrm{CO}$ and ${ }^{13} \mathrm{CO}$ fluctuations obtained via integrating the intensity over the entire emission line for L1512 molecular cloud. Counter-intuitively, Stutzki et al. (1998) found for both isotopes the power spectrum with a similar spectral index. According to LP04 this may correspond to optically thick assymptotics (i.e. the integration range of velocities is larger than $V_{c}$ (see Eq. 7)). If the velocity fluctuations dominate, the expected index is universal and equal to -3 (meaning $K^{-3}$ ), if the density fluctuations dominate (see Table 2) the expected index is $-3+\gamma$ (meaning $K^{-3+\gamma}$ ). The index measured in Stutzki et al. (1998) is $\sim 2.8$, which may either correspond to -3 within the experimental errors, or more likely indicate the that $\gamma \approx 0.2$, i.e. the density spectrum is shallow. The latter possibility is indirectly supported by ${ }^{18} \mathrm{CO}$ measurement for L1551 cloud in Swift (2006), used the VCA (observing the changes of the channel map spectral index while changing the velocity slice thickness) and obtained the shallow density spectrum with $\gamma \approx 0.2$, while his measured velocity spectrum was approximately Kolmogorov (the index is -3.72). Padoan et al. (2006) both successfully tested the VCA with high resolution numerical simulations that included radiative transfer and applied the technique to Five College Radioastronomy Observatory (FCRAO) survey of the Perseus molecular cloud complex. He obtained the velocity index around -3.81 .

VCS predictions were successfully tested in Chepurnov \& Lazarian (2006b). In Chepurnov \& Lazarian (in preparation) the technique was applied to the Arecibo high latitude galactic data. Figure 4 illustrates the results obtained for maps with high spectral resolution as well as for the spatially smoothed maps with lower spatial resolution. This allowed to study both the high and low resolution regimes of the VCS. The results obtained for the aforementioned regimes are similar, namely, the power spectrum indexes are around -3.87 , which is somewhat steeper than the Kolmogorov index.

The practical application of the VCA and the VCS has only started. Nevertheless, it

10 In the case of an isobaric medium the product of density and temperature are constant and the problem
is similar to studies of transitions for which the emissivity is proportional to $\rho^{2}$ that we discussed earlier. 
has already provided some intriguing results and proved to be a promising way of using the wealth of spectroscopic surveys for studies of astrophysical turbulence.

Acknowledgments I thank Alexey Chepurnov, Dmitry Pogosyan and Snezana Stanimirovic for their input. AL research is supported by by NSF grant AST 0307869 and the NSF Center for Magnetic Self Organization in Laboratory and Astrophysical Plasmas.

\section{REFERENCES}

1. Armstrong, J. W., Rickett, B. J., \& Spangler, S. R. ApJ, 443, 209 (1995)

2. Ballesteros-Paredes, J. Klessen, R., Mac Low, M., Vazquez-Semadeni, E. astro-ph/0603357

3. Begum, A., Chengalur, J., \& Bharadwaj, S. MNRAS Letters in press, astro-ph/0607367(2006)

4. Beresnyak, A., Lazarian, A., \& Cho, J. ApJ Letters, 624, 93 (2005)

5. Chepurnov, A. \& Lazarian, A. ApJ, submitted (2006ab)

6. Cho, J., Lazarian, A. MNRAS, 345, 325 (2003)

7. Deshpande, A.A., Dwarakanath, K.S., Goss, W.M., ApJ, 543, 227 (2000)

8. Dickey, J., McClure-Griffiths, N., Stanimirovic, S., Gaensler, B., \& Green, A. ApJ, 561, 264 (2001)

9. Falgarone, E, \& Puget, J.-L. A\& A, 293, 840 (1995)

10. Falgarone, E., Pineau Des Gorets, B., Hily-Blant, P., \& Schilke, P. astro-ph/0606222 (2006)

11. Green, D.A. MNRAS, 262, 328 (1993)

12. Goldreich, P., \& Sridhar, S. ApJ, 438, 763 (1995)

13. Hartmann, L., \& MacGregor, K. B. ApJ, 242, 260 (1980)

14. Heiles, C. \& Troland, T. ApJS, 151, 271

15. Heyer, M., \& Brunt, C. ApJ, 615, 45 (2004)

16. Elmegreen, B. ApJ, 577, 206 (2002)

17. Elmegreen, B. \& Falgarone, E. ApJ, 471, 816 (1996)

18. Elmegreen, B. \& Scalo, J. ARA\&A, 42, 211 (2004)

19. Ensslin, T. \& Vogt, C. A\&A, 453, 447 (2006)

20. Esquivel, A. \& Lazarian, A. ApJ, 784, 320 (2005)

21. Esquivel, A., Lazarian, A., Pogosyan, D., Cho, J. MNRAS, 342, 325 (2003)

22. Khalil, A, Joncas, G., Nekka, F., Kestener, P., \& Arneodo, A. ApJS, 165, 512 (2006)

23. Lazarian, A. ApJ Letters, 645, L25 (2006a)

24. Lazarian, A. AN, 327, 609 (2006b)

25. Lazarian, A. JKAS, 37, 563 (2004)

26. Lazarian, A. \& Esquivel, A. ApJ Letters, 592, L37 (2003)

27. Lazarian, A. \& Pogosyan, D. ApJ, 537, 720 (2000)

28. Lazarian, A. \& Pogosyan, D. ApJ, 616, 943 (2004)

29. Lazarian, A. \& Pogosyan, D. ApJ, in press, astro-ph/0511248 (2006)

30. Lazarian, A., Pogosyan, D., Vazquez-Semadeni, E., \& Pichardo, B. ApJ, 555, 130 (2001)

31. Lazarian, A., Vishniac, E., \& Cho, J. ApJ, 603, 180 (2004)

32. Levier, F. $A \& A, \mathbf{4 2 1}, 387$ (2004)

33. Mac Low, M. \& Klessen, R. RvMP, 76, 125 (2004)

34. McKee, C., Tan, J., Nature, 416, 59 (2002)

35. McKee, C. "The Dynamical Structure and Evolution of Giant Molecular Clouds" in The Origin of Stars and Planetary Systems, ed. Charles J. Lada and Nikolaos D. Kylafis. Kluwer, 1999 p.29

36. Miville-Deschênes, M., Levrier, F., \& Falgarone, E. A\&A, 593, 831 (2003)

37. Muller, E., Stanimirovic, S., Rosolowsky, E., Staveley-Smith, L. ApJ, 616, 845

38. Munch, G. Rev. Mod. Phys., 30, 1035 (1958)

39. Padoan, P, Juvla, M., Kritsuk, A., \& Norman, M. ApJ, submitted

40. Padoan, P., Goodman, A., \& Juvela, M. ApJ, 588, 881 (2003)

41. Ossenkopf, V., Esquivel, A., Lazarian, A. \& Stutzki, J. A\&A, 452, 223

42. Shu, F., Li, Z.-Y., \& Allen, A. ApJ, 601, 930 (2004)

43. Stanimirovic, S. \& Lazarian, A. ApJ, 551, L53 (2001)

44. Stutzki, J., Bensch, F., Heithausen, A., Ossenkopf, V., \& Zielinsky, M. A\&A, 336, 697 (1998) 
45. Spangler, S., \& Gwinn, C. ApJ, 353, L29 (1990)

46. Sunyaev, R.A., Norman, M.L., \& Bryan, G.L. Astronomy Letters, 29, 783 (2003)

47. Swift, J. PhD Thesis, Univ. of California-Berkeley (2006) 